Appendix B

Stimuli Used in Experiment 2

Appendix C

Stimuli Used in Experiment 3

\begin{tabular}{lll}
\hline Regular & Strange & Exception \\
\hline part & High Frequency & \\
time & friend & done \\
not & front & give \\
still & earth & both \\
life & once & some \\
held & two & have \\
stop & his & shall \\
each & school & says \\
just & their & put \\
take & who & does \\
name & piece & said \\
help & young & great \\
with & group & were \\
or & from & are \\
page & world & do \\
& she & what \\
plump & Low Frequency & \\
carve & laugh & gross \\
yell & bulb & steak \\
rink & juice & deaf \\
cub & guy & spook \\
hunt & tongue & pint \\
gate & debt & wool \\
tent & worse & broad \\
pest & earn & lose \\
oak & comb & choose \\
stuff & view & sew \\
sock & yolk & doll \\
truck & sleigh & worm \\
soap & seize & touch \\
smile & ghost & shoe \\
& choir & wash \\
\hline & & \\
& & \\
& & \\
& &
\end{tabular}

\begin{tabular}{lll}
\hline Regular & Strange & Exception \\
\hline thin & High Frequency & \\
least & earth & watch \\
nine & piece & choose \\
race & sign & touch \\
these & view & break \\
face & knife & some \\
beach & eye & says \\
shell & friend & wool \\
wake & once & lose \\
still & ghost & wash \\
feel & two & doll \\
corn & climb & give \\
& tongue & heard \\
mode & Low Frequency & \\
fern & gauge & deaf \\
pest & sword & tomb \\
math & seize & steak \\
hike & chute & soot \\
chore & heir & worm \\
greed & aisle & sew \\
grill & brooch & phase \\
dock & tsar & gross \\
bakes & corps & plaid \\
tile & sieve & wan \\
rust & choir & caste \\
& weird & wand \\
\hline
\end{tabular}

(Manuscript received September 1, 1983 revision accepted for publication March 3, 1984.)

\title{
NOTICES
}

\section{Search for Editor of Memory \& Cognition}

The new editor is expected to begin handling submissions about January 1, 1985, or shortly thereafter. Nominations of qualified individuals should be sent to Robert G. Crowder, Department of Psychology, Yale University, Box 11A Yale Station, New Haven, CT 06520, by August $1,1984$.

25th Annual Meeting of The Psychonomic Society, Inc.-Change of Dates for Meeting

The dates for the 25th annual meeting of The Psychonomic Society, Inc., have been changed:

$$
\begin{aligned}
& \text { From-November 1-3, } 1984 \\
& \text { To-November 8-10, } 1984
\end{aligned}
$$

The meeting still will be held at the Hilton Palacio Del Rio, San Antonio, Texas. 